\title{
DIFFUSION PARAMETER CONTROL OF SPATIOTEMPORAL CHAOS
}

\author{
RAUL MONTAGNE,* \\ Instituto de Física, Facultad de Ciencias \& Facultad de Ingeniería, \\ T. Narvaja 1674, C. P. 11200, Universidad de la República, Montevideo, Uruguay. \\ Departament de Física, Universitat de les Illes Balears, \\ E-07071 Palma de Mallorca, Spain.
}

\author{
PERE COLET, ${ }^{\dagger}$ \\ Instituto Mediterráneo de Estudios Avanzados, IMEDEA (CSIC-UIB) \\ E-07071 Palma de Mallorca, Spain.
}

Submitted to Int. J. Bifurcation and Chaos, 31 Agost 1997

\begin{abstract}
The stabilization of periodic solutions in the regime of spatiotemporal chaos through a diffusion parameter control is studied. We show that unstable plane waves in the Complex Ginzburg-Landau equation can be effectively stabilized in chaotic regimes such as phase turbulence and spatiotemporal intermittency or defect turbulence.
\end{abstract}

\section{Introduction}

There have been recently a considerable experimental and theoretical effort to characterize Spatiotemporal Chaos (STC) [Cross \& Hohenberg, 1994; Gollub, 1994]. Weak STC seems to be an ubiquitous phenomenon in large non-equilibrium systems. In some cases STC arise in the proximity of threshold and can be described within the context of weakly nonlinear theories. These theories are well developed in the form of so-called complex Ginzburg-Landau equations (CGLE) [Cross \& Hohenberg, 1993]. The CGLE is a prototypical equation for a complex field $A$ that exhibit STC [Chaté, 1995]. It accounts for the slow modulations, in space and time of the oscillatory state in a physical system which undergoes a Hopf bifurcation [van Saarloos \& Hohenberg, 1992].

The control of spatiotemporal chaos is a complicated problem, and so, there is a wide variety of methods intended to control such chaotic behavior. There have been several attempts to achieve such control in the CGLE [Aranson et al., 1994; Bleich \& Socolar, 1996; Mertens et al., 1994; Battogtokh \& Mikhailov, 1996; Montagne \& Colet, 1997]. The most common approach is adding time-delayed feedback terms to the CGLE. The feedback can be either local [Bleich \& Socolar, 1996] (at each spatial point, the field at the same point at previous times is fed back) or global [Mertens et al., 1994; Battogtokh \& Mikhailov, 1996] (at each spatial point a term proportional to the integral of the field over the spatial variable is fed back). Feedback has also been used for control in a nonlinear drift-wave equation driven by a sinusoidal wave [Gang, 1993] and, in conjunction with a spatial filter, it has been applied to stabilize rolls and hexagonal structures in a model for a transversally extended three level laser [Lu et al., 1996] and to control filamentation in a model for wide aperture semiconductor lasers based on the Swift-Hohenberg equation [Bleich et al., 1997].

A different approach using nonlinear diffusion effects has been introduced recently in [Montagne \& Colet, 1997]. It was shown that an imaginary nonlinear diffusion term was able to stabilize unstable plane waves in several regimes of STC. Here we generalize this study considering the case of having both, real and imaginary nonlinear diffusion terms in the CGLE. One of the main advantages of this generalization is the possibility of controlling plane waves outside the region where control works using only an imaginary nonlinear diffusion term. In particular, in that case it was practically impossible to stabilize plane waves with a large wavevector, whereas this can be easily achieved in the case shown here. Also, in situations where control was already possible, this extension allows to achieve the stabilization of plane waves using a control parameter with 
the control techniques, stabilization is possible only if starting from an initial state close to the unstable orbit. If the initial condition is an arbitrary chaotic state, the system will explore the different regions of the chaotic attractor in phase space and eventually may approach the unstable orbit one wishes to stabilize although this can take an extremely long time. If the system goes close enough to the unstable orbit then the control technique shown here should work. Although we are not exploring in detail how close to the unstable orbit the system has to be to achieve stabilization, we show numerically that our scheme is robust to finite size perturbations of the initial condition.

In section 2 we briefly describe the parameter regions for which different chaotic behaviors have been found for the CGLE and we introduce the modified equation. Section 3 is devoted to the linear stability analysis of the plane wave solutions. We calculate for which parameter values the added term is able to stabilize plane waves in the STC regions of the CGLE. In section 4 we show integrating the equations numerically, that the region of stability of the plane waves when finite size perturbations are applied is in excellent agreement with the analytical prediction of the linear stability analysis. Finally we give some concluding remarks in Section 5.

\section{Model}

The one dimensional CGLE [van Saarloos \& Hohenberg, 1992; Cross \& Hohenberg, 1993; van Saarloos, 1995; Newell et al., 1993] for a complex field $A(x, t)$, describes the slow dynamics of spatially extended systems close to a Hopf bifurcation,

$$
\partial_{t} A=A+\left(1+i c_{1}\right) \partial_{x}^{2} A-\left(1+i c_{2}\right)|A|^{2} A .
$$

We will assume periodic boundary conditions through all the paper. This equation admits among other exact solutions, plane waves of the form

$$
A_{p w}(x, t)=A_{0} e^{i(k x-\omega t)},
$$

with amplitude $A_{0}=\sqrt{1-k^{2}},|k|<1$ and frequency $\omega=c_{2}+\left(c_{1}-c_{2}\right) k^{2}$.

For $1+c_{1} c_{2}>0$ plane wave solution are linearly stable for wave numbers smaller than a limit value $|k| \leq k_{E}$ given by

$$
k_{E}^{2}=\frac{1+c_{1} c_{2}}{3+c_{1} c_{2}+2 c_{2}^{2}} .
$$

For $|k|>k_{E}$, plane waves are unstable to phase perturbations (Eckhaus instability [Eckhaus, 1965]). The stability range vanishes at $1+c_{1} c_{2}=0$, the Benjamin-Feir-Newell (BFN) line, and there are no stable plane wave solutions for $1+c_{1} c_{2}<0$.

Numerical work for large system size [Shraiman et al., 1992; Chaté, 1994; Chaté, 1995] has identi- fied regions of the parameter space displaying different kinds of regular and spatiotemporal chaotic behavior, leading to a "phase diagram" for the CGLE in the plane $c_{1}-c_{2}$. The five different regions, each leading to a different asymptotic phase, are shown in Fig. 1. Two of these regions are in the BFN stable zone and the other three in the BFN unstable one. In this paper we will concentrate in the regimes with a chaotic behavior, namely SpatioTemporal Intermittency, Phase Turbulence and Defect Turbulence. A detailed description can be found in [Chaté, 1995].

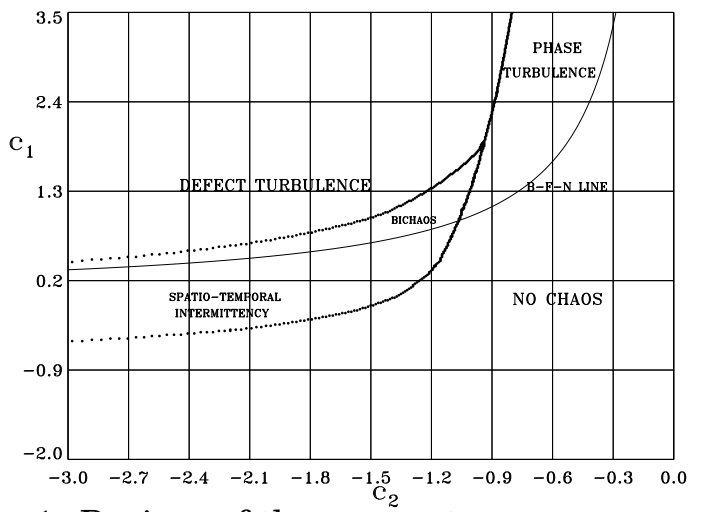

Figure 1: Regions of the parameter space $c_{1}-c_{2}$ for which the $d=1$ CGLE displaying different kinds of regular and chaotic behavior. The Benjamin-FeirNewell line (BFN line) is also shown.

We modify the CGLE by changing a parameter of the system dynamically and proportionally to the deviation of the system from the state to be stabilized. We will show that stabilization of plane waves can be achieved by replacing the coefficient $c_{1}$ by $c_{1}+$ $\gamma\left(|A|^{2} /\left|A_{p w}\right|^{2}-1\right)$ where $\gamma=\gamma_{r}+i \gamma_{i}$ is a complex constant and $\left|A_{p w}\right|=A_{0}$ is the modulus of the plane wave to be stabilized. Notice that as the added term $\gamma\left(|A|^{2} / A_{0}^{2}-1\right)$ vanishes identically for $A=A_{0}$, any plane wave $A_{0}$ that is a solution of (1) is also a solution of the modified equation. We are not changing the solution but we will change its stability. The added term also preserves the phase invariance of the solution of the original CGLE, $A \rightarrow A e^{i \psi}$, with $\psi$ being an arbitrary phase. The modified CGLE is then explicitly given by,

$\partial_{t} A=A+\left[1+i c_{1}+\gamma\left(|A|^{2} / A_{0}^{2}-1\right)\right] \partial_{x}^{2} A-\left(1+i c_{2}\right)|A|^{2} A$.

\section{Stability Analysis}

We use a standard linearization procedure for studying the stability of the plane wave solutions (2) of eq. (4) . Consider the time evolution of small perturbations in the amplitude and phase,

$$
A(x, t)=\left(A_{0}+\epsilon r(x, t)\right) e^{i(k x-\omega t+\epsilon \phi(x, t))},
$$

where $r(x, t)$ and $\phi(x, t)$ are real perturbations in the amplitude and phase respectively and $\epsilon$ is a formal 
parameter to keep track of small numbers.

Substituting (5) in (4) yields to a polynomial in $\epsilon$. The first order terms yield the linearized equations for the perturbations.

$$
\begin{aligned}
\partial_{t} r & =-2\left(\gamma_{r} k^{2}+A_{0}^{2}\right) r-2 A_{0} k \partial_{x} \phi-2 c_{1} k \partial_{x} r \\
& -c_{1} A_{0} \partial_{x}^{2} \phi+\partial_{x}^{2} r \\
\partial_{t} \phi & =-2 c_{2} A_{0} r-2 \gamma_{i} \frac{k^{2}}{A_{0}} r-2 c_{1} k \partial_{x} \phi+2 \frac{k}{A_{0}} \partial_{x} r \\
& +\partial_{x}^{2} \phi+\frac{c_{1}}{A_{0}} \partial_{x}^{2} r
\end{aligned}
$$

We consider solutions of (6) and (7) proportional to $e^{\eta t+i q x}$, where for periodic boundary conditions $q$ is real whereas $\eta$ is in general a complex quantity. By substituting in (6) and (7) we obtain the dispersion relation

$\left|\begin{array}{cc}\eta+2 A_{0}^{2}+2 \gamma_{r} k^{2}+q^{2}+2 i c_{1} q k & 2 i q k-c_{1} q^{2} \\ c_{1} q^{2}+2 c_{2} A_{0}^{2}-2 i q k+2 \gamma_{i} k^{2} & \eta+q^{2}+2 i c_{1} q k\end{array}\right|=0$.

The solutions of (8) are

$$
\eta=-\left(A_{0}^{2}+\gamma_{r} k^{2}+q^{2}+2 i c_{1} q k\right) \pm \sqrt{u+i v},
$$

where $u$ and $v$ are polynomials

$$
\begin{aligned}
u & =\left(A_{0}^{2}+\gamma_{r} k^{2}\right)^{2}+4 q^{2} k^{2}-2 c_{1} c_{2} A_{0}^{2} q^{2}-c_{1}^{2} q^{4} \\
& -2 \gamma_{i} c_{1} q^{2} k^{2} \\
v & =4 q k\left(c_{1} q^{2}+c_{2} A_{0}^{2}+\gamma_{i} k^{2}\right) .
\end{aligned}
$$

The real part of $\eta$ indicates the growth rate of the perturbations,

$$
R e(\eta)=-A_{0}^{2}-\gamma_{r} k^{2}-q^{2} \pm \sqrt{\frac{u+\sqrt{u^{2}+v^{2}}}{2}}
$$

The plus and minus sign $( \pm)$ correspond to two different branches. For the negative sign in (12), $\operatorname{Re}(\eta)<$ 0 , so these perturbations are always damped.

For the positive sign of the square root in (12) $R e(\eta)=0$ at $q=0$, so all the plane wave solutions are marginally stable. The origin of this neutral stability is the phase invariance $A \rightarrow A e^{i \psi}$ of the solutions of the equations (1) and (4). For $q$ very large, $R e(\eta) \simeq-q^{2}$, so short wavelength perturbations are always damped. However long wavelength perturbations can grow destabilizing the original plane wave solution, to see this we expand (12) for small $q$.

$$
R e(\eta)=D q^{2}+O\left(q^{4}\right)
$$

where

$$
\begin{aligned}
D & =-1-\frac{c_{1} c_{2} A_{0}^{2}}{a}+2\left(1+\frac{c_{2}^{2} A_{0}^{4}}{a^{2}}\right) \frac{k^{2}}{a} \\
& +\gamma_{i}\left(-\frac{c_{1} k^{2}}{a}+4 \frac{c_{2} k^{4} A_{0}^{2}}{a^{3}}\right)+2 \gamma_{i}^{2} \frac{k^{6}}{a^{3}} .
\end{aligned}
$$

and

$$
a=\left(A_{0}^{2}+\gamma_{r} k^{2}\right)
$$

If this coefficient is positive, there is a range of long wavelength perturbations that grow. The condition $D<0$ is necessary for stability but not sufficient, since the growth coefficient obtained from the full expression (12) can be positive for some $q$ despite the coefficient $D$ being negative. However, for the values of $c_{1}$ and $c_{2}$ considered in this work the requirement $D<0$ gives a very good limit for the stability region.

For the unperturbed CGLE $(\gamma=0)$ the condition $D<0$, leads to the standard Eckhaus instability limit: $|k|<k_{E}$ with $k_{E}$ given by (3). Since the control is done through a diffusive term, the added term never changes the stability of the homogeneous solution $k=0$. For an arbitrary plane wave of wave number $k$ one has to solve the cubic equation $D=0$ to find explicitly the range of values of $k$ for which the plane wave is stable. In Figs. 2 - 3 we have plot this range as a function of the parameter $c_{1}$ for several values of $\gamma$ and $c_{2}$. When no control is applied $(\gamma=0)$ the stability region in the $k-c_{1}$ plane is limited by a branch of eq. (3) (dashed line in Figs. 2 - 3) whose vertex corresponds to the BFN point. Decreasing the value of $c_{1}$ the width of the stability region $|k|<k_{E}$ increases and for $c_{1} \rightarrow-\infty, k_{E} \rightarrow 1$.

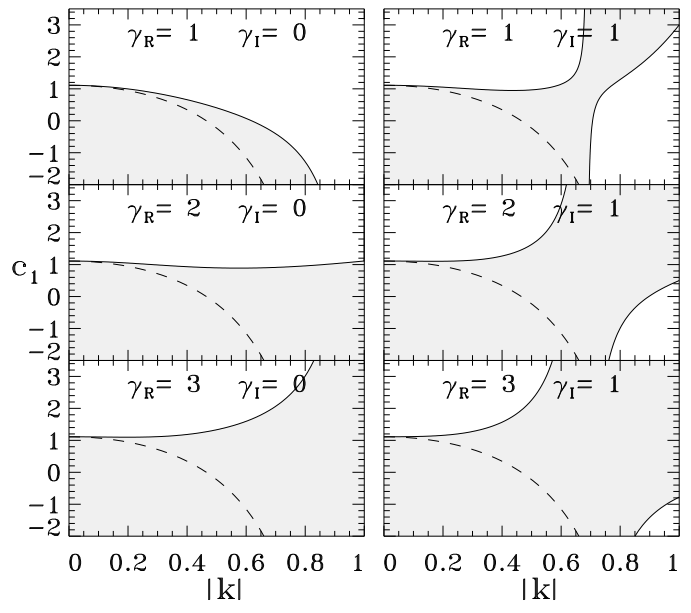

Figure 2: The shadowed zone shows the stability region for the plane wave (2) for $c_{2}=-0.9$. Left column and from up to bottom , $\gamma_{i}=0$ and $\gamma_{r}=1,2,3$. Left column and from up to bottom, $\gamma_{i}=1$ and $\gamma_{r}=1,2,3$. For comparison, the boundary of the stability region for $\gamma=0$, given by Eq. (3), is shown in all the figures as a dashed line.

As $|\gamma|$ is increased the stability region changes its shape, so that it is possible to stabilize plane waves which were unstable without control. Increasing the value of $|\gamma|$ one finds stabilization of plane waves for values of $c_{1}$ well above the BFN line, in the regions of phase turbulence and defect turbulence of the original CGLE. In particular, for $c_{2}=-0.9$ the BFN line corresponds in Fig. 2 to an horizontal line at $c_{1}=1.11$. Below this value for $c_{1}$, plane waves with a 
ble without control. As shown in Fig. 2 they can be easily stabilized with a real control parameter. For $c_{1}>1.11$ we are in the phase turbulence regime up to $c_{1} \approx 2.3$ (see Fig. 1). For larger values of $c_{1}$ we enter in the defect turbulence region. It is possible to stabilize plane waves in the region of phase turbulence taking $\gamma_{r} \geq 3$ and $\gamma_{i}=0$, or $\gamma_{r} \geq 1$ and $\gamma_{i}=1$. For $c_{2}=-2.1$ the BFN line corresponds in (Fig. 3) to an horizontal line at $c_{1}=0.476$. Below that level there are the coexistence of stable plane waves and spatiotemporal intermitency. Plane waves with wavevector outside the dashed line are unstable and will lead to spatio-temporal intermitency without control. As shown in Fig. 3 the real part of $\gamma$ is very effective in the stabilization of these plane waves. For $c_{1}>0.476$ we are above the BFN line, and increasing $c_{1}$ we enter first in the bichaos regime and later in the defect turbulence regime (see Fig. 1). As shown in Fig. 3, stabilization of plane waves in both regions is possible using for example $\gamma=1+i$.

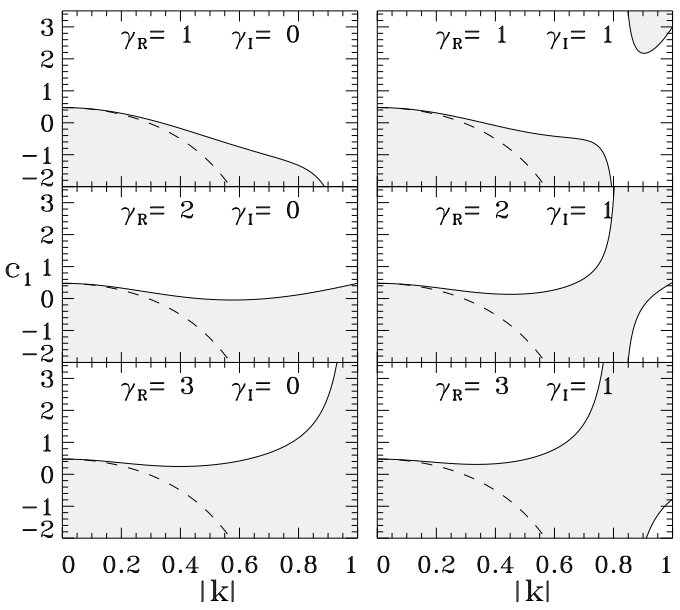

Figure 3: The shadowed zone shows the stability region for the plane wave (2) for $c_{2}=-2.1$. Left column and from up to bottom, $\gamma_{i}=0$ and $\gamma_{r}=1,2,3$. Left column and from up to bottom, $\gamma_{i}=1$ and $\gamma_{r}=1,2,3$. For comparison, the boundary of the stability region for $\gamma=0$ is shown in all the figures as a dashed line.

In general, the real part of $\gamma$ is specially effective in extending the stability region to plane waves of large wave number. We should stress that stabilization of plane waves with wavevector close to one was extremely difficult to achieve using only an imaginary nonlinear diffusion term, even using large values for $\gamma_{i}$, as shown in [Montagne \& Colet, 1997]. Above the BFN line, stabilization of unstable plane waves in the regions of phase and defect turbulence can be achieved taking $\gamma$ to be purely real (left column of Figures 2 and 3) or purely imaginary (see ref. [Montagne \& Colet, 1997]). However, it is more efficient to take $\gamma$ complex, since one can achieve stabilization with smaller values of $|\gamma|$.

\section{Numerical simulations}

We have integrated numerically the eq. (1) and (4) using a pseudospectral code with periodic boundary conditions and second-order accuracy in time. Spatial resolution was typically 1024 modes. Time step was typically $\Delta t=0.001$. The system size was always taken as $L=512$. The details of the numerical method can be seen in Ref. [Montagne et al., 1997]. We start from an initial condition which corresponds to a plane wave plus a small random perturbation

$$
A(x, t=0)=\sqrt{1-k^{2}} e^{i k x}+\sigma \xi(x)
$$

where $\xi(x)$ is a complex Gaussian random perturbation of zero mean and variance $\left\langle\xi(x) \xi^{*}\left(x^{\prime}\right)\right\rangle=2 \delta(x-$ $\left.x^{\prime}\right)$.

We have performed numerical simulations in different regions of the phase diagram (Fig. 1) to verify the results obtained from the linear stability analysis when finite size perturbations are applied. We have found a very good agreement between the prediction of the linear stability analysis and the numerical simulations. As characteristic examples we show the following results.

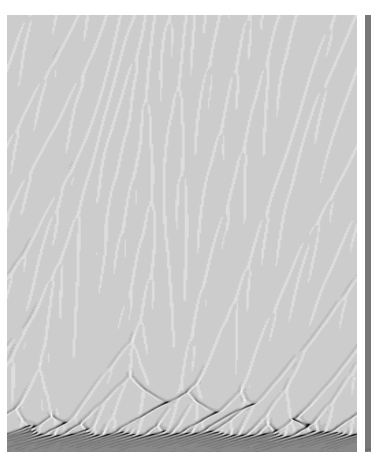

a)
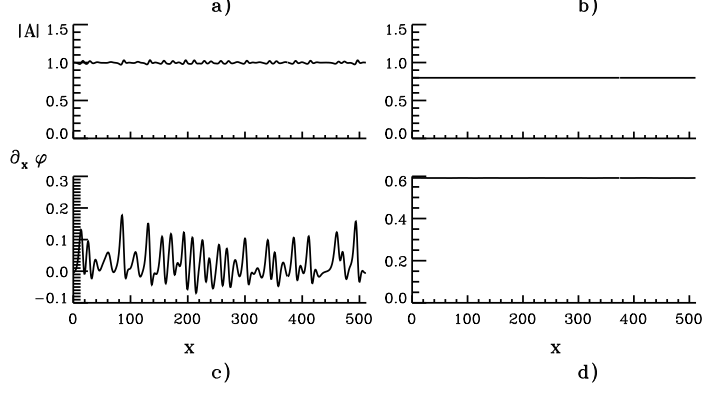

Figure 4: Spatiotemporal evolution of the CGLE (4) for $c_{1}=1.5, c_{2}=-0.9$ starting from a perturbed plane wave (16) with $k=0.5$ and $\sigma=0.007$. Figs. a) and b) show $|A(x, t)|$ with time running upward from $t=0$ to $t=1000$ and $\mathrm{x}$ in the horizontal direction for $\gamma=0$ and $\gamma=3+0 i$ respectively. The absolute value of the field $\left|A\left(x, t_{0}\right)\right|$ and the phase gradient $\partial_{x} \phi\left(x, t_{0}\right)$ at $t_{0}=950$ are displayed in c) and d) for $\gamma=0$ and $\gamma=3+0 i$ respectively. 
Fig. 4 shows the stabilization of a plane wave for parameter values $c_{1}=1.5$ and $c_{2}=-0.9$. This corresponds to the phase turbulence regime (see fig. 1 ), where no plane waves are stable for the original CGLE. As predicted by the linear stability analysis a perturbed plane wave with $k=0.5$ can easily be stabilized with $\gamma_{r}=3$ and $\gamma_{i}=0$ while for $\gamma=0$ the same initial condition decays in time $t=80$ (approx.) to phase turbulence.

Fig. 5, obtained for parameter values $c_{1}=2.5$ and $c_{2}=-2.1$, shows stabilization of plane waves in the region of defect turbulence, where for the unperturbed CGLE there are no stable plane waves and the field $A$ shows a strongly disordered STC state characterized by the presence of defects. As predicted by the linear stability analysis, a perturbed plane wave with $k=0.9$ can be stabilized with $\gamma_{r}=1$ and $\gamma_{i}=1$.
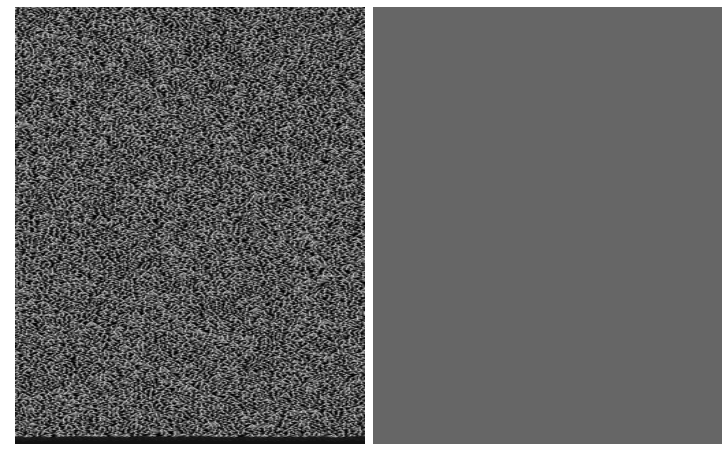

b)
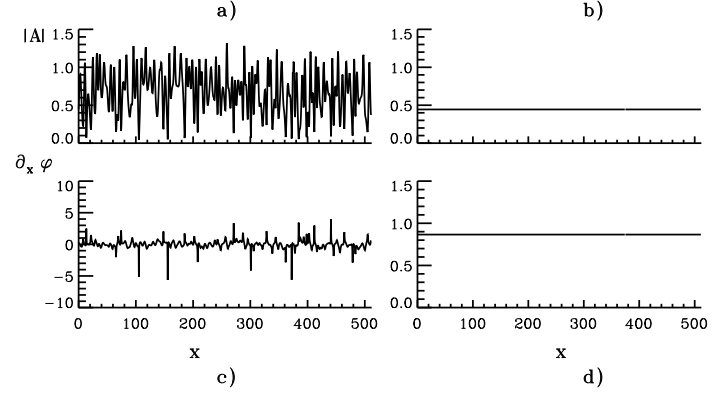

Figure 5: Spatiotemporal evolution of the CGLE for $c_{1}=2.5, c_{2}=-2.1$ starting from a perturbed plane wave with $k=0.9$. Figs. a) and b) show $|A(x, t)|$ for $\gamma=0$ and $\gamma=1+i$ respectively. The values of $\left|A\left(x, t_{0}\right)\right|$ and $\partial_{x} \phi(x, t)$ at $t_{0}=950$ are displayed in $\left.\mathrm{c}\right)$ and d) for $\gamma=0$ and $\gamma=1+i$ respectively. Other parameters are as in Fig. 4

\section{Concluding Remarks}

Our study of the dynamics of the CGLE with a nonlinear diffusion term demonstrates the possibility of stabilization of an unstable plane wave in different regimes of STC. Since the added term vanish for the stabilized plane wave, this plane waves are exactly the same unstable solutions of the original CGLE.
Although our method does not change the stability of the homogeneous solution $k=0$, it is quite effective in stabilizing plane waves with non zero wave vector. We have shown that the real part of the nonlinear diffusive term is specially effective for stabilizing plane waves with wavevector close to one. Also, in general it is more efficient to use a nonlinear diffusive term with both real and imaginary parts, in the sense that stabilization can be achieved with a control parameter with smaller absolute value.

Finally, we have studied numerically the stability of the plane waves when finite size perturbations are applied. The results are in excellent agreement with the analytical predictions of the linear stability analysis.

\section{Acknowledgements}

Financial support from Dirección General de Investigación Científica y Técnica (DGICYT, Spain), Projects PB94-1167 and PB94-1172, is acknowledged. R.M. also acknowledges partial support from the Programa de Desarrollo de Ciencias Básicas (PEDECIBA, Uruguay).

\section{References}

Aranson, I., Levine, H. \& Tsimring, L. [1994] "Controlling spatiotemporal chaos," Phys. Rev. Lett. 72, 2561-2564.

Battogtokh, D. \& Mikhailov, A. [1996] "Controlling turbulence in the complex Ginzburg-Landau equation," Physica D 90, 84-95.

Bleich, M. \& Socolar, J. E. S. [1996] "Stability of periodic orbits controlled by time-delay feedback," Phys. Lett. A 210, 87-94.

Bleich, M. E., Hochheiser, D., Moloney, J. V. \& Socolar, J. E. S. [1997] "Controlling extended systems with spatially filtered, time-delayed feedback," Phys. Rev. E 55, 2119-2126.

Chaté, H. [1994] "Spatiotemporal intermittency regimes of the one-dimensional complex GinzburgLandau equation," Nonlinearity 7(1), 185-204.

Chaté, H. [1995] "Disordered regimes of the onedimensional complex Ginzburg-Landau equation," in Cladis, P. \& Palffy-Muhoray, P., editors, Spatiotemporal Patterns in Nonequilibrium Complex Systems, vol. XXI of Santa Fe Institute in the Sciences of Complexity, pp. 5-49 (Addison-Wesley) New York.

Cross, M. \& Hohenberg, P. [1993] "Pattern formation outside of equilibrium," Rev. Mod. Phys. 65, 851-1112. and references therein. 
Cross, M. \& Hohenberg, P. [1994] "Spatiotemporal chaos," Science 263, 1569-1570.

Eckhaus, W. [1965] Studies in nonlinear stability theory (Springer) Berlin.

Gang, H. [1993] "Controlling chaos in systems described by partial differential equations," Phys. Rev. Lett. 71, 3794-3797.

Gollub, J. [1994] "Spirals and chaos," Nature 367, 318.

Lu, W., Yu, D. \& Harrison, R. [1996] "Control of patterns in spatiotemporal chaos in optics," Phys. Rev. Lett. 76, 3316-3319.

Mertens, F., Imbihl, R. \& Mikhailov, A. [1994] "Turbulence and standing waves in oscillatory chemical reactions with global coupling," J. Chem. Phys. 101, 9903-9908.

Montagne, R. \& Colet, P. [1997] "Nonlinear diffusion control of spatiotemporal chaos in the complex Ginzburg-Landau equation," Phys. Rev. E 56, 40174024.
Montagne, R., Hernández-García, E., Amengual, A. \& San Miguel, M. [1997] "Wound-up phase turbulence in the Complex Ginzburg-Landau equation," Phys. Rev. E 56, 151-167.

Newell, A. C., Passot, T. \& Lega, J. [1993] "Order parameter equations for patterns," Annu. Rev. Fluid Mech. 25, 399-453.

Shraiman, B., Pumir, A., van Saarloos, W., Hohenberg, P., Chaté, H. \& Holen, M. [1992] "Spatiotemporal chaos in the one-dimensional complex Ginzburg-Landau equation," Physica D 57, 241248.

van Saarloos, W. [1995] "The complex GinzburgLandau equation for beginners," in Cladis, P. \& Palffy-Muhoray, P., editors, Spatiotemporal Patterns in Nonequilibrium Complex Systems, vol. XXI of Santa Fe Institute in the Sciences of Complexity, p. 19-31 (Addison-Wesley) New York.

van Saarloos, W. \& Hohenberg, P. [1992] "Fronts, pulses, sources and sinks in generalized complex Ginzburg-Landau equations," Physica D 56, 303367. and (Errata) Physica D 69, 209 (1993).

*Email montagne@fisica.edu.uy, WWW http://www.imedea.uib.es/ montagne

${ }^{\dagger}$ Email pere@hp1.uib.es, WWW http://www.imedea.uib.es/ pere 